\title{
Cognitive control of working memory but not familiarity in rhesus monkeys (Macaca mulatta)
}

\author{
Emily Kathryn Brown ${ }^{1} \cdot$ Robert R. Hampton ${ }^{1}$ \\ Published online: 7 July 2020 \\ (C) The Psychonomic Society, Inc. 2020
}

\begin{abstract}
Directed forgetting paradigms assess cognitive control by determining whether memory accuracy is superior in trials on which subjects were instructed to remember compared with accuracy in trials on which they were instructed to forget. We used a directed forgetting paradigm to compare the extent to which working memory and familiarity are subject to rehearsal-like cognitive control in rhesus monkeys. Monkeys studied a sample image, then saw one of two distinctive cues during a retention interval. The remember cue typically predicted a four-choice match to sample test, for which memory of the sample was critical. The forget cue typically predicted one of five perceptual discrimination tests, matched for accuracy to the memory tests, for which memory of the sample was irrelevant. On rare probe trials, the test type other than the type typically predicted by the cue was presented. When cognitive control of memory was possible, accuracy should have been higher on memory tests following the remember cue than on those following the forget cue. We found that accuracy was higher following the remember cue under conditions that favored working memory (small image set) but was not higher under conditions that favored matching on the basis of relative familiarity (large image set). Working memory, but not familiarity, is subject to cognitive control in rhesus monkeys.
\end{abstract}

Keywords Directed forgetting $\cdot$ Rehearsal $\cdot$ Primate cognition

\section{Introduction}

Memory results from multiple processes that differ in their accessibility to cognitive control. Working memory requires cognitive control (Baddeley, 2003). Performance in working memory tests suffers when subjects are distracted or required to engage in a competing cognitive task (e.g., Basile \& Hampton, 2013; Brady \& Hampton, 2018a; Clapp, Rubens, \& Gazzaley, 2010; Doherty et al., 2018; Hassett \& Hampton, 2017). Because only a limited amount of information can be simultaneously kept active in working memory (Cowan, 2001, 2010; Engle, 2001; Miller, 1956), terminating rehearsal of some information frees up limited cognitive resources for rehearsal of other information or other mental activities. Cognitive control can thereby optimize working memory by focusing rehearsal effort on the most relevant material. By contrast, some other forms of memory may not require, or

Emily Kathryn Brown

emily.brown@emory.edu

1 Department of Psychology and Yerkes National Primate Research Center, Emory University, 36 Eagle Row, Atlanta, GA 30322, USA be accessible to, active cognitive control. Familiarity signals are automatically evoked by seeing a previously viewed image (Yonelinas, 2002; Yonelinas, Aly, Wang, \& Koen, 2010). Accuracy in memory tests that can be solved by familiarity is affected little by distraction or competing cognitive load (Basile \& Hampton, 2013; Yonelinas \& Jacoby, 1994).

As is in humans, memory in non-humans is also comprised of multiple systems, as indicated by both behavioral and neurobiological evidence, and these systems may differ in the extent to which they are accessible to cognitive control (e.g., Packard \& McGaugh, 1996; Sherry \& Schacter, 1987; Squire \& Zola-Morgan, 1991; Tu, H.-W., Hampton, \& Murray, 2011). In monkeys, item recognition is supported by at least two types of memory: working memory and relative familiarity (Brady \& Hampton, 2018a; Wittig, Morgan, Masseau, \& Richmond, 2016; Wittig \& Richmond, 2014). Monkey working memory is vulnerable to competing cognitive load (Basile \& Hampton, 2013; Smith, Coutinho, Church, \& Beran, 2013) and information in monkey working memory can be selectively prioritized to enhance retention of the most relevant material (Brady \& Hampton, 2018b; Tu, H.-W. \& Hampton, 2014). The distinction between cognitively controlled and automatic memory processes has additionally been made across a variety 
of taxa using "process dissociation" paradigms that closely parallel findings with human subjects (Guitar \& Roberts, 2015; Jacoby, 1991; Roberts, Guitar, Marsh, \& MacDonald, 2016a; Roberts, Macpherson, \& Strang, 2016b; Roberts, Strang, \& Macpherson, 2015; Tu, H.-W. et al., 2011; Tu, H. W. \& Hampton, 2013). Here we used the converging operation of a directed forgetting paradigm to assess the reliability of previously reported dissociations of working memory and familiarity in monkeys.

Directed forgetting paradigms demonstrate the existence of cognitive control when subjects show better memory for material they have been instructed to remember compared to material they have been instructed to forget (e.g., Hourihan, Ozubko, \& MacLeod, 2009). To the extent that remembering a studied item depends on a memory system that is subject to cognitive control, active maintenance should occur when subjects have been cued to remember and should be attenuated when subjects are cued to forget (Sheard \& MacLeod, 2005). By contrast, if remembering a studied item depends on an automatic memory process that is not subject to cognitive control, the cue to remember or forget should not affect retention. Non-human directed forgetting tasks employ "remember" cues that predict a test of memory for a previously viewed sample and "forget" cues that predict a test unrelated to that sample. On occasional probe tests, the experimenter tests memory despite having cued the subject to forget. Roper and Zentall (1993) critiqued earlier studies of directed forgetting in non-humans (e.g.,Grant \& Barnet, 1991; Kendrick, Rilling, \& Stonebraker, 1981; Maki \& Hegvik, 1980), and identified a set of methodological concerns that must be addressed to confirm the phenomenon. Under conditions that addressed these concerns, monkeys demonstrated cognitive control: they were more accurate when cued to expect a memory test than when cued to expect a discrimination matched for probability of reinforcement (Tu, H.-W. \& Hampton, 2014). Therefore, the directed forgetting paradigm is well suited to dissociate memory systems in monkeys on the basis of whether or not the systems are subject to cognitive control.

If monkeys, like humans, use cognitive control to maintain working memory but detect familiarity automatically, then test conditions that encourage heavy reliance on working memory should generate stronger directed forgetting effects than do test conditions that favor matching on the basis of relative familiarity. The size of the pool of images used to generate memory tests has a powerful effect on the utility of relative familiarity as a mnemonic signal for discriminating samples from distractors (Brady \& Hampton, 2018a). When a small set of images is used repeatedly across trials, all of the images become familiar and proactive interference builds (Basile \& Hampton, 2010; Edhouse \& White, 1988; Jitsumori, Wright, \& Cook, 1988; Wright, Urcuioli, \& Sands, 1986). Because all images are highly familiar, it is difficult to identify the most recently seen sample image on the basis of relative familiarity.
Instead, subjects must rely on working memory to identify the sample image seen on the current trial. In contrast, when a large set of images is used to generate the samples and distractors for memory tests, the sample image is highly familiar relative to the distractors that appear with it at test, because the distractors have not been seen for a relatively long time. In such tests, the recently seen sample image can be readily identified by relative familiarity (Brady \& Hampton, 2018a)

In the current study, we used a directed forgetting procedure, combined with manipulation of image set size, to determine the relative accessibility of working memory and familiarity to cognitive control. We manipulated the utility of familiarity as a mnemonic signal, and thus the necessity of working memory for accurate recognition, across two blocked experimental phases. In the first block of trials we used a small set of repeating images to favor working memory. In the second block of trials, we used a large set of images that monkeys saw infrequently, maximizing the utility of familiarity as a mnemonic signal. If working memory, but not familiarity, is subject to cognitive control, we should observe a directed forgetting effect for images drawn from small, but not large, image sets. We also manipulated the duration of retention intervals to explore how memory strength affects directed forgetting.

\section{Methods}

\section{Subjects}

We used seven pair-housed male rhesus macaque monkeys (Macaca mulatta), average age 7 years at the beginning of these studies. Five monkeys had prior experience with a similar directed forgetting paradigm that used different "remember" and "forget" cues and a different discrimination task on "forget trials" (Tu \& Hampton 2014). All monkeys had extensive experience with cognitive testing.

\section{Apparatus}

We tested monkeys in their home cages, using portable touchscreen computer rigs consisting of a laptop computer (Dell, Round Rock, TX) with generic speakers, a 15-in. color LCD touchscreen (ELO, Menlo Park, CA, USA), and two automated food dispensers (Med Associates Inc., St. Albans, VT, USA) that dispensed into food cups beneath the screen. Food reinforcement consisted of 94 or $97 \mathrm{mg}$ of nutritionally complete primate pellets (Bio-Serv, Frenchtown, NJ, USA and Purina TestDiet, Richmond, IN, USA). We presented stimuli and collected responses using programs written in Presentation (Neurobehavioral Systems, Albany, CA, USA). Pellets earned during testing were subtracted from monkeys' 
daily primate biscuit chow rations, such that monkeys consumed roughly the same number of calories daily, regardless of testing performance. Daily calorie budgets were established by veterinary staff based on weight trajectories and clinical assessments.

\section{General task properties}

To start a trial, monkeys touched a green ready square at the bottom center of the screen. A sample image then appeared on the screen (Fig. 1). Touches to this image extinguished the sample and caused a memory cue to appear. On $50 \%$ of trials, the memory cue was the "remember" cue, a fermata notation. On the other $50 \%$ of trials, the memory cue was the "forget" cue, a not-equal symbol. To help ensure that monkeys attended to the memory cue, it was presented in randomly selected locations, and monkeys had to touch it to advance the trial. The cue then dimmed and remained onscreen throughout the delay so that monkeys did not have the increased memory load of remembering the memory cue. Typically, a match-to-sample memory test followed the "remember" cue. Also typically, a perceptual discrimination test followed the "forget" cue. On probe trials memory tests followed the "forget" cue and discrimination tests followed the "remember" cue. During training, the cues were valid and correctly predicted the task that would follow on $100 \%$ of trials; during testing with probe trials, cues were valid on $90 \%$ of trials.

Correct selection of the sample in memory tests, or the target stimulus in discrimination tests, resulted in distinctive auditory feedback and food reinforcement of one pellet. Incorrect selection of one of the distractors resulted in distinctive auditory feedback and the screen going blank for a 5-s time-out interval. All responses required two touches (FR2) to prevent registering undirected contacts to the touchscreen as choices.

\section{Match-to-sample memory tests}

Following the memory cue on match-to-sample tests, the sample stimulus seen at study and three distractors were presented in the four corners of the screen. First, monkeys completed all trials in the small image set condition, wherein the same four clip-art images were seen at test on every trial. After completing trials with the small set of images, monkeys completed all trials in the large stimulus set condition, wherein images were drawn without replacement from a pool of 1,600 photos of birds that had been converted into black-and-white line drawings (Fig. 2). Monkeys typically match with higher accuracy when tested with images drawn from a large, compared to a small, image set (e.g., Basile \& Hampton, 2010; Mishkin \& Delacour, 1975). In order to match accuracy with images drawn from the two set sizes, researchers have used longer

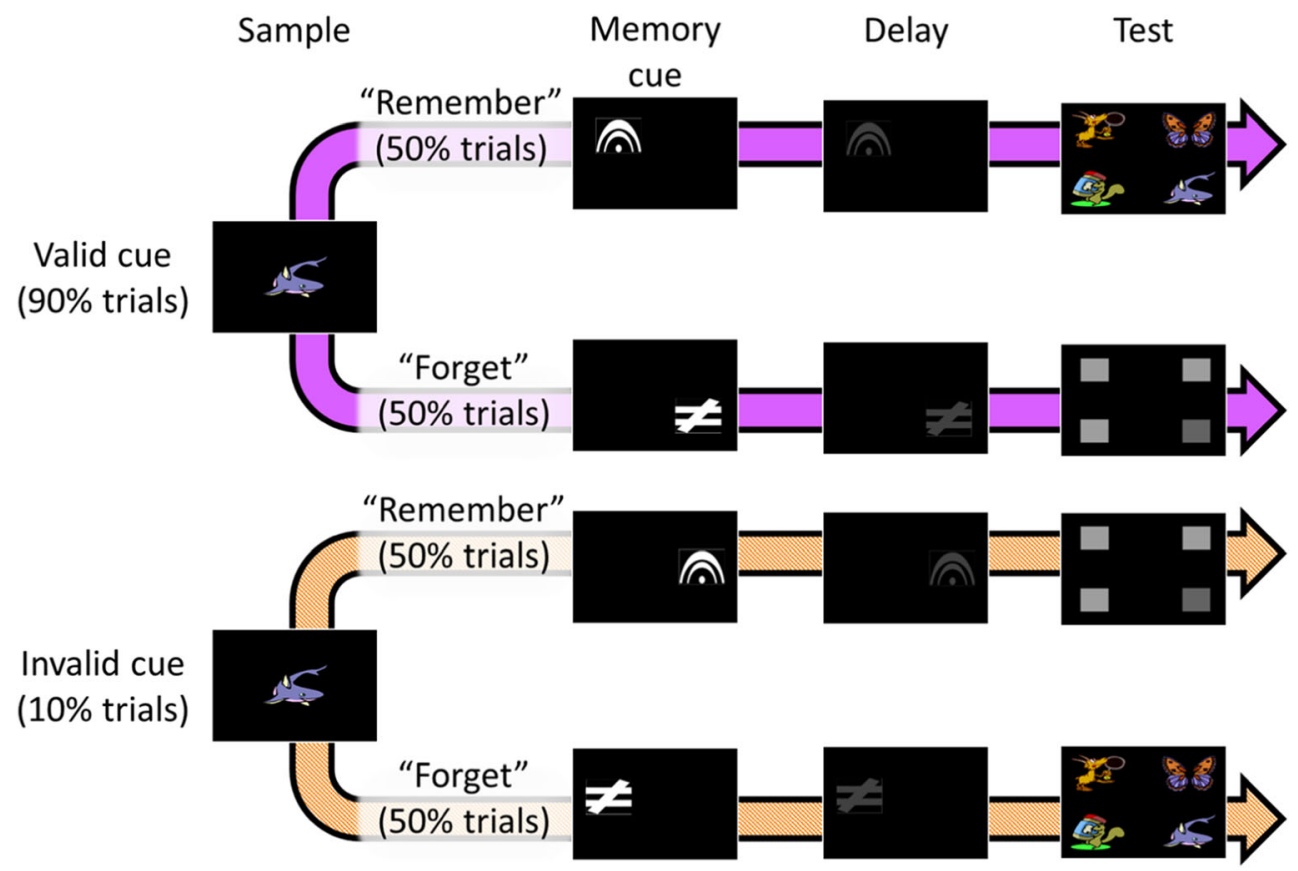

Fig. 1 Steps to complete a trial of the directed forgetting task. Monkeys touched a green square to initiate trials (not shown). After monkeys touched a sample image, a memory cue appeared. After training with $100 \%$ valid cue trials, monkeys progressed to probe sessions. The memory cue correctly predicted the type of test that would follow on $90 \%$ of trials (top). On $10 \%$ of probe session trials (bottom), the memory cue did not correctly predict the test type. Monkeys were cued to "remember" on half of trials and were cued to "forget" on the other half. The memory cue dimmed and remained onscreen during the delay. Correct selection of the sample on match-to-sample tests or the target item on perceptual discriminations resulted in food reinforcement 

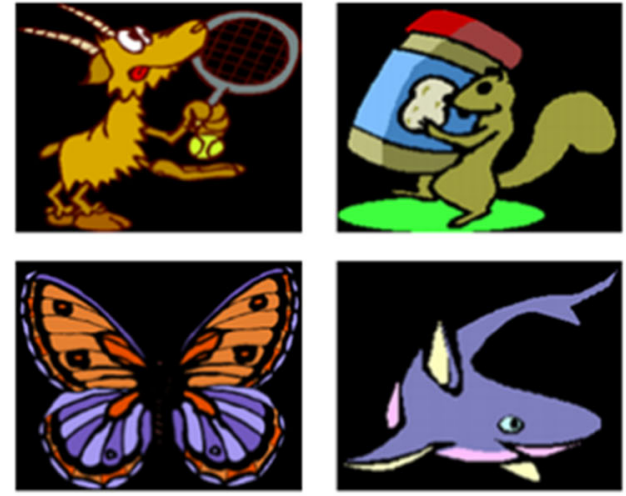

\section{Small stimulus set}

Fig. 2 Examples of the memory test stimuli that could follow valid "remember" cues or invalid "forget" cues. In the small image set condition, the same four clip-art images were seen on every trial; in the

delays for images from the large set (e.g., Basile \& Hampton, 2013). In addition to manipulating delay, we created a large set from perceptually similar images, because otherwise monkeys were accurate in memory tests with delays so long they prohibited testing.

\section{Discrimination tests}

Following the "forget" cue on discrimination trials, we presented a perceptual oddity discrimination that consisted of a target stimulus and three distractors that occupied each of the four corners of the screen. Within each discrimination test, the target stimulus remained the same across trials. Each discrimination consisted of two different distracter values, one lesser (e.g., dimmer) and one greater (e.g., brighter) in magnitude than the target by equal amounts. On a given trial, the three distractors were identical values to one another (e.g., all circles of the same smaller size than the target in the size discrimination task).

If monkeys learned to expect a specific discrimination test following "forget" cues, they might hold in working memory the correct response for the discrimination, in anticipation of the test. Holding this response in working memory might displace memory for the sample image seen at the beginning of the trial. To prevent such prospection, we pseudo-randomly selected among five different perceptual discriminations, preventing the monkeys from anticipating any one test (Tu \& Hampton, 2013; Washburn \& Astur, 1998). The perceptual tests used were an arc length discrimination, a circle size discrimination, a dot rotation discrimination, a brightness discrimination, and a dot density discrimination (Fig. 3).
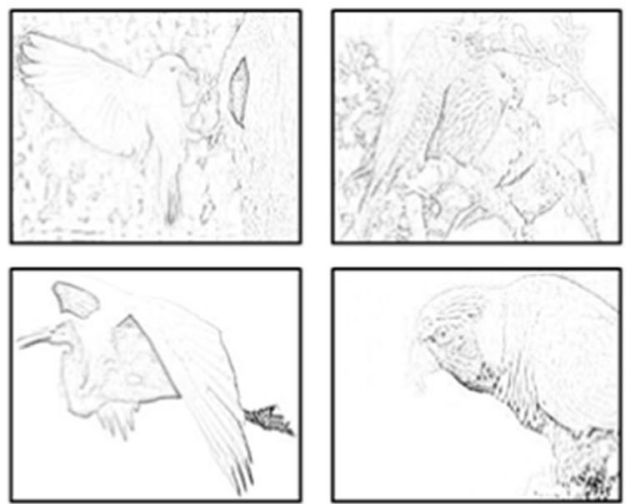

\section{Examples from \\ large stimulus set}

large image set condition, the images were drawn from a pool of 1,600 photos of birds that had been converted into black-and-white line drawings

\section{Training and probe trials}

During training, we titrated performance in the memory tests to achieve 50-70\% accuracy, where we expected monkeys to be far enough from ceiling and floor performance that a directed forgetting effect would be evident, if present. Monkeys were trained on match-to-sample with $100 \%$ valid remember cues, until they completed at least two sessions of the task in the criterion accuracy range. Monkeys were also trained on the five perceptual discriminations at intermixed difficulty levels until they were highly proficient with the easiest discriminanda. During this training, monkeys saw distractors from five different difficulty levels for each discrimination task, which ranged from easy (very dissimilar to the target) to hard (very similar to the target). The difficulty level for each discrimination that was closest to the center of the criterion accuracy range of $50-70 \%$ for a given monkey was selected for use in testing sessions that contained directed forgetting probe trials. After probe trials began, monkeys saw only their two criterion-level distractor sets and one target value for each discrimination in a given session. Thus, there were five target values (one correct response per discrimination) and ten distractor values (one incorrect value in each direction per discrimination) for discriminations for each monkey. During probe trials, discrimination difficulty and delay length were adjusted between sessions to maintain accuracy in the criterion range.

For probes, titrated delays for the small set condition ranged across monkeys from 3 to $120 \mathrm{~s}(3,14,18,18,21$, 24 , and $120 \mathrm{~s}$ ) and titrated delays for the large set condition ranged from 20 to $256 \mathrm{~s}(20,32,152,180,224,240$, and 256 s). We used these titrated delays to generate a range of delays tailored to each monkey. The "long" delays were double the 


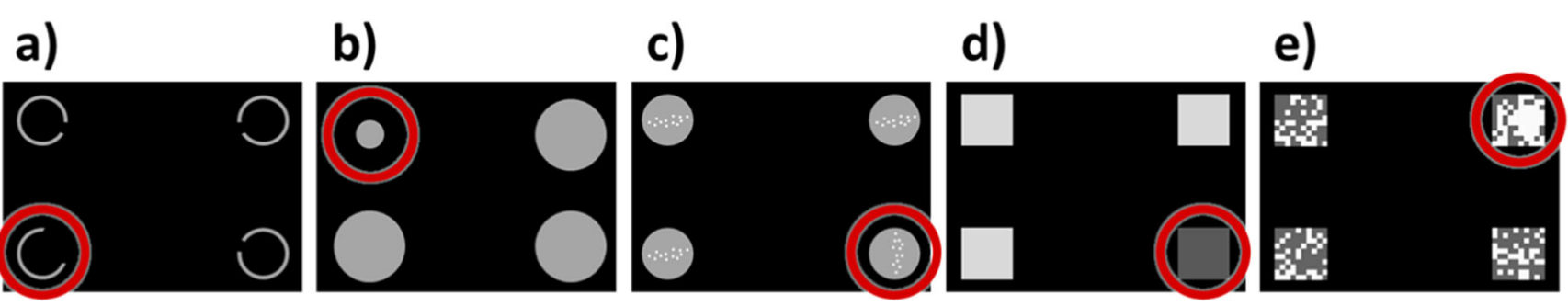

Fig. 3 Examples of the five perceptual discrimination tests that could follow valid "forget" cues or invalid "remember" cues. Perceptual discrimination tests required discrimination on the basis of (a) arc length, (b) circle size, (c) dot rotation, (d) brightness, and (e) dot density. The target (circled) was assigned randomly to the four corners of the screen. Examples shown here exaggerate the differences presented

titrated delay for a given monkey, and the "short" delays were half and one-quarter of the titrated delay. During probe sessions, the memory cue accurately predicted the test that would follow on $90 \%$ of trials, and inaccurately predicted the test that would follow on the remaining $10 \%$ of trials. For each set size condition, monkeys completed at least four 3,200-trial sessions to ensure that we had selected appropriate target stimuli and delays, of which the last session, which should most closely reflect asymptotic performance, was used for analysis.

\section{Data analysis}

All proportions were arcsine transformed before statistical analysis to better approximate the normality assumption underlying parametric statistics (Keppel \& Wickens, 2004, p. 155).

We used a linear mixed-effect model, with subject as random factor. Linear mixed-effect models are useful in studies with a small $\mathrm{N}$ because they make separate estimates of slope for each individual that can help to account for random variation, and because they handle changes in variability across repeated observations better than the standard ANOVA (Gueorguieva \& Krystal, 2004). The model used to analyze memory accuracy included the image set size (large or small), cue validity (valid or invalid), and retention interval (Delay/4, Delay/2, Titrated Delay, Delay $* 2$ ). The model used for follow-up tests within each image set size included cue validity and retention interval. Models were fitted using $\mathrm{R}$ Version 3.5.0 (R Core Team) and R package lmerTest (Kuznetsova, Brockhoff, \& Christensen, 2015). Satterthwaite approximations were used for significance levels and degrees of freedom.

We assessed accuracy by calculating the proportion correct for valid cue memory tests ("remember" cue followed by memory test), invalid cue memory tests ("forget" cue followed by memory test), valid cue discrimination tests ("forget" cue followed by discrimination test), and invalid cue discrimination tests ("remember" cue followed by discrimination test), at to monkeys to make the discriminations more perceptible to the reader. The distractors shown in this figure vary in only one direction from the standard target; in testing, distractors could vary in either direction from the target (e.g., either smaller than or larger than the target on a given trial). But in all cases the distractors presented on a given trial were identical values to one another

each of the four retention intervals (Delay/4, Delay/2, Delay, and Delay*2).

\section{Results}

Monkeys were more accurate on memory tests preceded by a valid "remember" cue only when remembering images drawn from the small image set: (Fig. 4; interaction of image set size and cue validity: $\left.F_{(1,90)}=11.94, P<.001\right)$. We further examined this interaction by conducting follow-up tests within each set size separately. Cue validity significantly affected accuracy for memory tests drawn from the small image set $\left(F_{(1,42)}=\right.$ $24.500, P<.001)$ but not for memory tests drawn from the large image set $\left(F_{(1,42)}=.383, P=.540\right)$. If rehearsal is more important the longer the delay, we should expect an interaction of cue validity and delay. We did not find an interaction of cue validity and delay in either the small image set $\left(F_{(3,42)}=.568\right.$, $P=.639)$ or the large image set $\left(F_{(3,42)}=.454, P=.716\right)$. Monkeys were significantly more accurate at short delays than long delays for memory tests drawn from both the small image set $\left(F_{(3,42)}=10.220, P<.001\right)$ and the large image set $\left(F_{(3,42)}\right.$ $=29.638, P<.001)$.

Monkeys were similarly accurate on discrimination tests preceded by valid and invalid "forget cues" cues (Fig. 5; $\left.F_{(1,90)}=.51, P=.476\right)$. Accuracy on discriminations was significantly different between set size conditions such that the monkeys were more accurate in the small set size condition $\left(F_{(1,90)}=46.35, P<.001\right)$. Monkeys gained expertise on the perceptual discriminations with time. Because we trained and tested set size conditions sequentially in a blocked design, we had to re-titrate the difficulty of discriminations to keep them in the correct accuracy range. However, because discrimination accuracy was in the target range in both conditions, we do not expect it to have contributed to differences in the directed forgetting effect. Accuracy on discriminations was also significantly affected by delay length such that the monkeys were less accurate at longer delays $\left(F_{(3,90)}=3.191, P\right.$ $=.027)$, possibly indicating that motivation or attention to 
Small Set Memory Accuracy

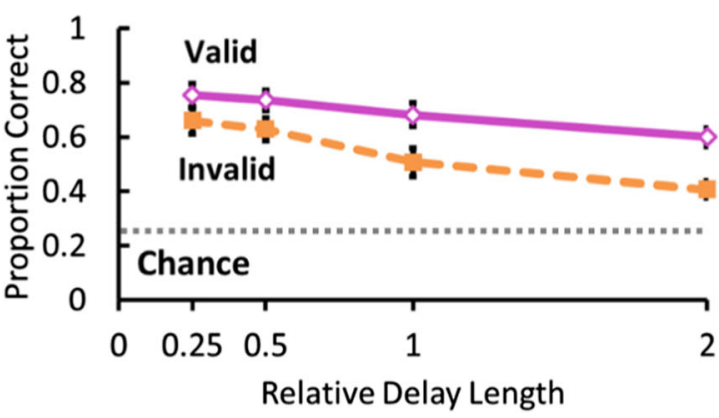

Fig. 4 Monkeys showed cognitive control with the small set (left) but not the large set of images (right). Mean accuracy is shown as the proportion correct \pm SEM across each of four delays. Tests were preceded by either a

accurate task completion waned with long delays, although this is a very small effect compared to that observed in memory tests.

\section{Discussion}

We found that working memory, but not familiarity, was subject to cognitive control in rhesus monkeys. In tests with a small set of repeating images, for which relative familiarity is a poor signal for discriminating recently seen samples from distractors, working memory is necessary to discriminate recently seen samples from distractors in memory tests (Basile \& Hampton, 2013; Brady \& Hampton, 2018a). Under these conditions we found that monkeys were more accurate on trials in which they were cued to remember than on those in which they were cued to forget, indicating active cognitive control. We did not find this difference when we tested monkeys with a large set of images, for which relative familiarity is a powerful mnemonic signal and working memory is of less value and possibly more difficult to engage (Basile \& Hampton, 2013; Brady \& Hampton, 2018a). Roberts et al. (1984) previously suggested that a small set of images might be a necessary condition for obtaining a directed forgetting

\section{Small Set Discrimination Accuracy}

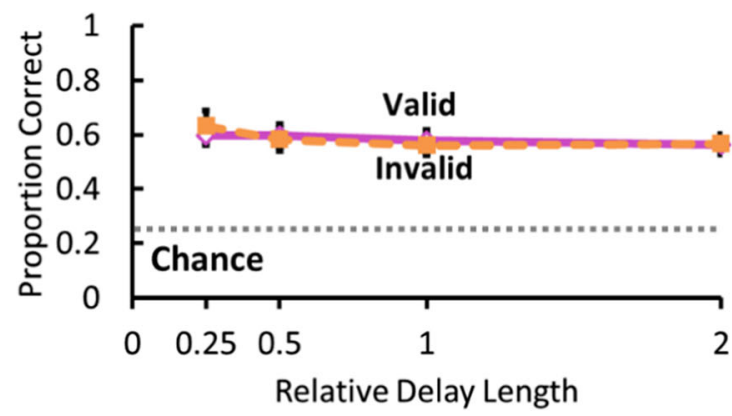

Fig. 5 Monkeys were similarly accurate on discrimination tests regardless of the validity of the "forget" cue in both the small set (left) and large set (right) conditions. Mean accuracy is shown as the
Large Set Memory Accuracy

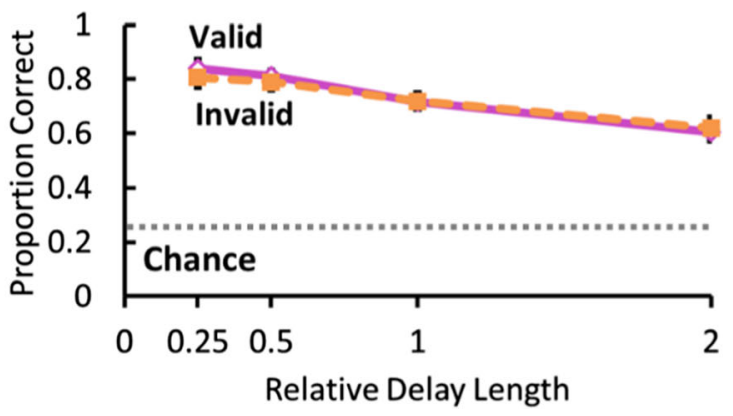

valid cue to remember (purple solid) or an invalid cue to forget (orange dashed). Dotted lines represent chance

effect. These findings show that the contents of working memory, but not familiarity signals, are subject to cognitive control during the delay interval.

Our dissociation of cognitive control of working memory and familiarity is consistent with a model of recognition memory performance that describes performance as the result of contributions from at least two memory systems: working memory and familiarity. Working memory depends on active maintenance of information about the to-be-remembered sample image in mind through a process like rehearsal. In contrast, familiarity is a passive and automatic process by which the correct image is identified based on relative familiarity at test (Basile \& Hampton, 2013; Brady \& Hampton, 2018a; Wittig et al., 2016; Wittig \& Richmond, 2014). By manipulating the size of the set from which images were drawn, we manipulated the degree to which working memory, and maintenance via cognitive control, was engaged. When test images were drawn from a relatively small set of repeating images, every image had been seen recently and was highly familiar, making familiarity a poor basis on which to identify the most recently seen image. In contrast, when test images were drawn from a large set of infrequently repeating images, the sample was easy to identify on the basis of relative familiarity at test, without active maintenance of information through the delay interval.

\section{Large Set Discrimination Accuracy}

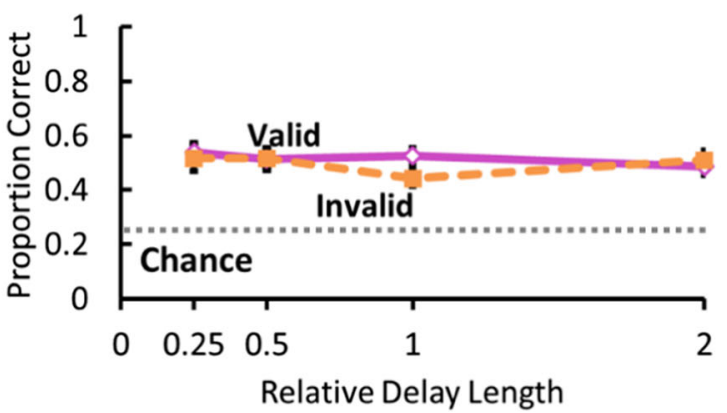

proportion correct \pm SEM across each of four delays. Tests were preceded by either a valid cue to remember (purple solid) or an invalid cue to forget (orange dashed). Dotted lines represent chance 
The set size conditions were blocked and all monkeys were tested with the small set size first and the large set size second. Therefore, we cannot entirely rule out the possibility that the differences in performance between the two image set size conditions were related to the order of testing. However, it seems less likely that highly trained animals would stop engaging in controlled rehearsal when doing so gave them the opportunity to increase their probability of food reward. Titrated delays in the large image set condition tended to be longer than in the small image set condition; however, because the range of tested delays overlapped between the two conditions, it is unlikely that delay length alone was responsible for the difference in directed forgetting effect between these two conditions. In the small set condition, the directed forgetting effect appears most evident at longer delays, which overlapped in range with the shortest delays in the large set condition.

We observed an effect of the "remember" cue only in the small set condition. But this does not necessitate the conclusion that working memory was not, or could not, be engaged by images from the large set (Brady \& Hampton, 2018a). In the large set condition, we would expect that monkeys are able to select the correct response on test on the basis of either working memory or familiarity. In the small set condition, the correct response must be based on working memory because relative familiarity is an unreliable means to choose among frequently repeated images. Thus, even if monkeys terminate rehearsal after a "forget" cue in the large set condition, the addition of familiarity may buffer recognition performance at test.

Several facts favor the hypothesis that the images in the large set did not engage working memory substantially. Because we titrated recognition accuracy to a moderate level, monkeys could have increased their accuracy in the large set condition with working memory, if it were available. Additional support for this interpretation comes from a previous study in which monkeys were required to remember items drawn from lists (Basile \& Hampton, 2010). Monkeys showed robust primacy when remembering list items drawn from small but not large sets of images, suggesting an advantage for rehearsing familiar items. However, Brady and Hampton (2018a) found evidence that unfamiliar images did weakly engage working memory in monkeys. Thus, it is likely that it is comparatively difficult, though not impossible, for monkeys to hold unfamiliar images in working memory. Alternatively, it is possible that it was the higher perceptual similarity of the black-and-white line drawings in the large set condition compared to the clip-art images in the small set condition that made them difficult to maintain in working memory. However, we note that Hourihan et al. (2009) found a directed forgetting effect in humans for highly visually similar black and white symbols, even when subjects were prevented from using verbal rehearsal. Even if monkeys were able to rehearse unfamiliar items drawn from large image sets, it seems the longer delays used here might be particularly challenging. Although the ranges of delays in the two image set conditions overlapped, much of the other evidence of $\operatorname{cog}$ nitively effortful working memory comes from experiments that use delays under $30 \mathrm{~s}$ in length, largely shorter than the delays used in the large set condition here (e.g., Basile \& Hampton, 2013; Brady \& Hampton, 2018a, 2018b; Brown, Basile, Templer, \& Hampton, 2019; Tu \& Hampton, 2014).

We did not find that cognitive control of working memory was significantly greater at long delays, which is inconsistent with the hypothesis put forward by Maki and Hegvik (1980) that longer delays would make successful remembering more dependent on cognitive control. Of the monkeys we tested, five individuals appeared to show some trend toward a larger directed forgetting effect at longer delays in the small set condition and two did not. This could indicate that five of the monkeys tended to rely more on working memory compared to the other two. Memory for unrehearsed items should deteriorate with the passage of time. If monkeys cannot sustain rehearsal at longer durations, their memory for "remember" cued items would therefore be lower at these intervals than for monkeys more effective at rehearsing. One future course of study might be to investigate whether different monkeys rely on different mnemonic strategies.

It is widely thought that cognitive control and cognitive monitoring work together to optimize cognitive processing (Nelson, 1996; Tu, Pani, \& Hampton, 2015). It is likely that cognitive control of working memory is possible during the delay because the memoranda are actively "held in mind," a process made possible by dynamic feedback from cognitive monitoring. Cognitive monitoring is a process that allows subjects to assess the strength, quality, or presence of a memory. According to this model, monkeys would assess their memory with cognitive monitoring and then "refresh" it as necessary with cognitive control. In contrast, familiarity can only be elicited once the test images are presented. Because familiarity is a memory signal elicited by the re-presentation of previously seen items, it cannot operate during the retention interval, when no images are present. Thus, monkeys might cognitively monitor familiarity signals if given the opportunity to do so at test, but the current work did not evaluate this possibility.

We used a directed forgetting paradigm to evaluate the extent to which working memory and familiarity are subject to cognitive control in monkeys. We found a directed forgetting effect only for images drawn from small sets, under conditions that we expected to favor use of working memory. We did not find a directed forgetting effect for images drawn from large sets, under conditions that we expected to favor familiarity. Our results suggest that monkeys, like humans, possess a working memory system that is subject to active cognitive control and a familiarity system that is not, drawing new 
parallels between human and non-human primate memory systems.

Author's Note This work was supported by the National Science Foundation (grants BCS-1632477, IOS-1146316, BCS-0745573) and the National Institutes of Health (grant RO1MH082819; T32HD071845). This project was supported in part by ORIP/OD P51OD011132.

We thank Jessica A. Joiner and Tara A. Dove-VanWormer for assistance with testing animals.

Data availability The datasets from the current study are available from the corresponding author on reasonable request.

\section{References}

Baddeley, A. D. (2003). Working Memory: Looking Back and Looking Forward. Nature Reviews Neuroscience, 4(10), 829-839.

Basile, B. M., \& Hampton, R. R. (2010). Rhesus Monkeys (Macaca Mulatta) Show Robust Primacy and Recency in Memory for Lists from Small, but Not Large, Image Sets. Behavioural Processes, 83(2), 183-190.

Basile, B. M., \& Hampton, R. R. (2013). Dissociation of Active Working Memory and Passive Recognition in Rhesus Monkeys. Cognition, 126(3), 391-396.

Brady, R. J., \& Hampton, R. R. (2018a). Nonverbal Working Memory for Novel Images in Rhesus Monkeys. Current Biology, 28(24), 39033910.e3.

Brady, R. J., \& Hampton, R. R. (2018b). Post-Encoding Control of Working Memory Enhances Processing of Relevant Information in Rhesus Monkeys (Macaca Mulatta). Cognition, 175, 26-35.

Brown, E. K., Basile, B. M., Templer, V. L., \& Hampton, R. R. (2019). Dissociation of Memory Signals for Metamemory in Rhesus Monkeys (Macaca Mulatta). Animal Cognition, 22(3), 331-341. doi:https://doi.org/10.1007/s10071-019-01246-5

Clapp, W. C., Rubens, M. T., \& Gazzaley, A. (2010). Mechanisms of Working Memory Disruption by External Interference. Cerebral Cortex, 20(4), 859-872.

Cowan, N. (2001). The Magical Number 4 in Short-Term Memory: A Reconsideration of Mental Storage Capacity. Behavioral and Brain Sciences, 24(1), 87-114.

Cowan, N. (2010). The Magical Mystery Four: How Is Working Memory Capacity Limited, and Why? Current Directions in Psychological Science, 19(1), 51-57.

Doherty, J. M., Belletier, C., Rhodes, S., Jaroslawska, A., Barrouillet, P., Camos, V., ... Logie, R. H. (2018). Dual-Task Costs in Working Memory: An Adversarial Collaboration. Journal of Experimental Psychology: Learning, Memory, and Cognition.

Edhouse, W. V., \& White, K. G. (1988). Cumulative Proactive Interference in Animal Memory. Animal Learning \& Behavior, 16(4), 461-467.

Engle, R. W. (2001). What Is Working Memory Capacity? In I. H. L. Roed-iger, J. S. Nairne, I. Neath, \& A. M. Surprenant (Ed.), The Nature of Remembering: Essays in Honor of Robert G. Crowder (pp. 297-314). Washington, DC: American Psychological Association Press.

Grant, D. S., \& Barnet, R. C. (1991). Irrelevance of Sample Stimuli and Directed Forgetting in Pigeons. Journal of the Experimental Analysis of Behavior, 55(1), 97-108.

Gueorguieva, R., \& Krystal, J. H. (2004). Move over Anova: Progress in Analyzing Repeated-Measures Data Andits Reflection in Papers
Published in the Archives of General Psychiatry. Archives of General Psychiatry, 61(3), 310-317.

Guitar, N. A., \& Roberts, W. A. (2015). The Interaction between Working and Reference Spatial Memories in Rats on a Radial Maze. Behavioural Processes, 112, 100-107.

Hassett, T. C., \& Hampton, R. R. (2017). Change in the Relative Contributions of Habit and Working Memory Facilitates Serial Reversal Learning Expertise in Rhesus Monkeys. Animal Cognition, 20(3), 485-497.

Hourihan, K. L., Ozubko, J. D., \& MacLeod, C. M. (2009). Directed Forgetting of Visual Symbols: Evidence for Nonverbal Selective Rehearsal. Memory \& Cognition, 37(8), 1059-1068. doi:https:// doi.org/10.3758/mc.37.8.1059

Jacoby, L. L. (1991). A Process Dissociation Framework: Separating Automatic from Intentional Uses of Memory. Journal of Memory and Language, 30(5), 513-541.

Jitsumori, M., Wright, A. A., \& Cook, R. G. (1988). Long-Term Proactive Interference and Novelty Enhancement Effects in Monkey List Memory. Journal of Experimental Psychology: Animal Behavior Processes, 14(2), 146.

Kendrick, D. F., Rilling, M., \& Stonebraker, T. B. (1981). Stimulus Control of Delayed Matching in Pigeons: Directed Forgetting. Journal of the Experimental Analysis of Behavior, 36(2), 241-251.

Keppel, G., \& Wickens, T. D. (2004). Design and analysis: a researcher's handbook. Prentice Hall.

Kuznetsova, A., Brockhoff, P. B., \& Christensen, R. H. B. (2015). Package 'Imertest'. R package version, 2(0).

Maki, W. S., \& Hegvik, D. K. (1980). Directed Forgetting in Pigeons. Animal Learning \& Behavior, 8(4), 567-574.

Miller, G. A. (1956). The Magical Number Seven, Plus or Minus Two: Some Limits on Our Capacity for Processing Information. Psychological Review, 63(2), 81.

Mishkin, M., \& Delacour, J. (1975). An Analysis of Short-Term Visual Memory in the Monkey. Journal of Experimental Psychology: Animal Behavior Processes, 1(4), 326.

Nelson, T. O. (1996). Consciousness and Metacognition. American Psychologist, 51(2), 102-116.

Packard, M. G., \& McGaugh, J. L. (1996). Inactivation of Hippocampus or Caudate Nucleus with Lidocaine Differentially Affects Expression of Place and Response Learning. Neurobiology of Learning and Memory, 65(1), 65-72.

Roberts, W. A., Mazmanian, D. S., \& Kraemer, P. J. (1984). Directed Forgetting in Monkeys. Animal Learning \& Behavior, 12(1), 29-40.

Roberts, W. A., Strang, C., \& Macpherson, K. (2015). Memory Systems Interaction in the Pigeon: Working and Reference Memory. Journal of Experimental Psychology: Animal Learning and Cognition, 41(2), 152.

Roberts, W. A., Guitar, N. A., Marsh, H. L., \& MacDonald, H. (2016a). Memory Systems in the Rat: Effects of Reward Probability, Context, and Congruency between Working and Reference Memory. Animal Cognition, 19(3), 593-604.

Roberts, W. A., Macpherson, K., \& Strang, C. (2016b). Context Controls Access to Working and Reference Memory in the Pigeon (Columba Livia). Journal of the Experimental Analysis of Behavior, 105(1), 184-193.

Roper, K. L., \& Zentall, T. R. (1993). Directed Forgetting in Animals. Psychological Bulletin, 113(3), 513-532. doi:https://doi.org/10. 1037/0033-2909.113.3.513

Sheard, E. D., \& MacLeod, C. M. (2005). List Method Directed Forgetting: Return of the Selective Rehearsal Account Dynamic Cognitive Processes (pp. 219-248): Springer.

Sherry, D. F., \& Schacter, D. L. (1987). The Evolution of Multiple Memory-Systems. Psychological Review, 94(4), 439-454.

Smith, J. D., Coutinho, M. V., Church, B. A., \& Beran, M. J. (2013). Executive-Attentional Uncertainty Responses by Rhesus Macaques 
(Macaca Mulatta). Journal of Experimental Psychology: General, 142(2), 458.

Squire, L. R., \& Zola-Morgan, S. (1991). The Medial Temporal-Lobe Memory System. Science, 253(5026), 1380-1386.

Tu, H. W., \& Hampton, R. R. (2013). One-Trial Memory and Habit Contribute Independently to Matching-to-Sample Performance in Rhesus Monkeys (Macaca Mulatta). Journal of Comparative Psychology, 127(3), 319-328. doi:https://doi.org/10.1037/a0030496

Tu, H.-W., \& Hampton, R. R. (2014). Control of Working Memory in Rhesus Monkeys (Macaca Mulatta). Journal of Experimental Psychology: Animal Learning and Cognition, 40(4), 467.

Tu, H.-W., Hampton, R. R., \& Murray, E. A. (2011). Perirhinal Cortex Removal Dissociates Two Memory Systems in Matching-to-Sample Performance in Rhesus Monkeys. The Journal of Neuroscience, 31(45), 16336-16343.

Tu, H.-W., Pani, A. A., \& Hampton, R. R. (2015). Rhesus Monkeys (Macaca Mulatta) Adaptively Adjust Information Seeking in Response to Information Accumulated. Journal of Comparative Psychology, 129(4), 347.

Washburn, D. A., \& Astur, R. S. (1998). Nonverbal Working Memory of Humans and Monkeys: Rehearsal in the Sketchpad? Memory \& Cognition, 26(2), 277-286.

Wittig, J. H., \& Richmond, B. J. (2014). Monkeys Rely on Recency of Stimulus Repetition When Solving Short-Term Memory Tasks. Learning \& Memory, 21(6), 325-333.
Wittig, J. H., Morgan, B., Masseau, E., \& Richmond, B. J. (2016). Humans and Monkeys Use Different Strategies to Solve the Same Short-Term Memory Tasks. Learning \& Memory, 23(11), 644-647.

Wright, A. A., Urcuioli, R. J., \& Sands, S. F. (1986). Proactive Interference in Animal Memory. In D. F. Kendrick, M. Riling, \& R. Denny (Eds.), Theories of Animal Memory (pp. 101-125). Hillsdale, NJ: Earlbaum.

Yonelinas, A. P. (2002). The Nature of Recollection and Familiarity: A Review of 30 Years of Research. Journal of Memory and Language, 46(3), 441-517.

Yonelinas, A. P., \& Jacoby, L. L. (1994). Dissociations of Processes in Recognition Memory: Effects of Interference and of Response Speed. Canadian Journal of Experimental Psychology/Revue canadienne de psychologie expérimentale, 48(4), 516.

Yonelinas, A. P., Aly, M., Wang, W. C., \& Koen, J. D. (2010). Recollection and Familiarity: Examining Controversial Assumptions and New Directions. Hippocampus, 20(11), 11781194

Publisher's note Springer Nature remains neutral with regard to jurisdictional claims in published maps and institutional affiliations. 